\title{
On the Propagation of Singularities of the Solution of the Cauchy Problem
}

\author{
By \\ Yûsaku HAMADA*
}

In this paper we consider the Cauchy problem for linear partial differential equation with holomorphic coefficients in complex domain.

We treat the case where the initial surface is non-characteristic and the initial data have singularities along a regular surface on the initial surface.

In $[2]$ we showed that, under the assumption that the characteristic surfaces issuing from the points of singularity in the initial data are simple and do not touch one another, the singularity in the initial data is propagated along these surfaces and if the initial data have at most poles (resp. essential singularities), the solution in general has at most poles (resp. essential singularities) and logarithmic singularities on these surfaces.

However, if an equation admits multiple characteristics, the behavior of the solution is different from that of the case of simple characteristics. For example we consider the Cauchy problem

$$
\left\{\begin{array}{l}
\frac{\partial^{2} u}{\partial x^{2}}-\frac{\partial u}{\partial y}=0 \\
u(0, y)=\frac{1}{y} \\
\frac{\partial u}{\partial x}(0, y)=0
\end{array}\right.
$$

The solution $u(x, y)$ is expressed in the form

Received June 30, 1970.

Communicated by S. Matsuura.

* Department of Mathematics, Kyoto Technical University. 


$$
u(x, y)=\frac{1}{y}+\frac{1}{y} \sum_{n=1}^{\infty}(-1)^{n} \frac{n !}{(2 n) !}\left(\frac{x^{2}}{y}\right)^{n}
$$

Thus, this example shows that although the initial data have pole, the solution has essential singularity on the surface $y=0$ which is double characteristic.

However, if we impose a suitable condition on the lower order terms of the differential equation, the behavior of the solution is similar to that of the case of simple characteristics. Now we impose E. E. Levi's condition on the differential operator. Let us recall that this condition was imposed in the studies on the well-posedness for weakly hyperbolic equation (cf. A. Lax [3], Mizohata-Ohya [8] and Matsuura [5]).

In this paper we shall give some results on these situations.

In the same way as [2], our method relies essentially on the papers of S. Mizohata [6] and [7]. We also refer to the results of D. Ludwig [4] and Gårding, Kotake, Leray [1].

In the next section, we shall give the precise statement of our results.

I wish to express my sincere gratitude to Professor S. Mizohata for his valuable advice and constant encouragement.

\section{$\S 1$. Notations and Results}

Let $x=\left(x_{1}, \ldots, x_{n}\right)$ denote the point in the space of $n$-complex variables $C^{n}$. We also write $x=\left(x_{1}, x^{\prime}\right), x^{\prime}=\left(x_{2}, \ldots, x_{n}\right)$ and $x^{\prime \prime}=\left(x_{3}, \ldots, x_{n}\right)$. Let $a\left(x, \frac{\partial}{\partial x}\right)$ be a linear partial differential operator of the form

$$
a\left(x, \frac{\partial}{\partial x}\right)=\sum_{|\alpha| \leq m} a_{\alpha}(x) \frac{\partial^{|\alpha|}}{\partial x_{1}^{\alpha_{1}} \ldots \partial x_{n}^{\alpha_{n}}}
$$

where $\alpha=\left(\alpha_{1}, \ldots, \alpha_{n}\right)$ is $n$-tuples of non-negative integers, $|\alpha|=\alpha_{1}+\cdots+\alpha_{n}$.

The coefficients $a_{\alpha}(x)$ are supposed to be holomorphic in a neighborhood of $x=0$.

We associate $a\left(x, \frac{\partial}{\partial x}\right)$ with the polynomial $a(x, \zeta)$ in the complex variables $\zeta=\left(\zeta_{1}, \ldots, \zeta_{n}\right)$ defined by 


$$
a(x, \zeta)=\sum_{|\alpha| \leq m} a_{\alpha}(x) \zeta^{\alpha} \quad\left(\zeta^{\alpha}=\zeta_{1}^{\alpha_{1}} \ldots \zeta_{n}^{\alpha}{ }^{\alpha}\right),
$$

and denote by $P_{k}(x, \zeta)$ its homogeneous part of degree $k$ :

$$
P_{k}(x, \zeta)=\sum_{|\alpha|=k} a_{a}(x) \zeta^{\alpha},
$$

then we have

$$
a(x, \zeta)=P_{m}(x, \zeta)+P_{m-1}(x, \zeta)+\cdots+P_{0}(x, \zeta) .
$$

In this paper we shall assume throughout that $a_{m, 0, \ldots, 0}(x) \equiv 1$, which implies that the surface $x_{1}=0$ is non-characteristic for $P_{m}\left(x, \frac{\partial}{\partial x}\right)$.

Our purpose is to consider the Cauchy problem:

$$
\begin{aligned}
& a\left(x, \frac{\partial}{\partial x}\right) u(x)=0 \\
& \frac{\partial^{k} u}{\partial x_{1}^{k}}\left(0, x^{\prime}\right)=w_{k}\left(x^{\prime}\right) \quad \text { for } \quad k=0,1, \ldots, m-1,
\end{aligned}
$$

where the initial data $w_{k}\left(x^{\prime}\right)(k=0,1, \cdots, m-1)$ are holomorphic in a neighborhood of $x^{\prime}=0$ except on the surface $x_{2}=0$.

In treating this problem, we shall impose on the differential operator $a\left(x, \frac{\partial}{\partial x}\right)$ the conditions due to Mizohata-Ohya $[8]$ and Matsuura [5]. That is, at first we require that the characteristic surfaces issuing from the points of singularity of the initial data are at most double and their multiplicities are independent of $x$.

In reality, we require more restrictive condition:

Condition (A). The decomposition

$$
P_{m}(x, \zeta)=\prod_{i=1}^{l_{1}}\left(\zeta_{1}-\lambda_{i}\left(x, \zeta^{\prime}\right)\right)^{2} \prod_{j=1}^{l_{2}}\left(\zeta_{1}-\mu_{j}\left(x, \zeta^{\prime}\right)\right)
$$

holds for all $x$ and $\zeta^{\prime}$ in a neighborhood of $x=0$ and $\zeta^{\prime}=(1,0, \ldots, 0)$. Here $\lambda_{i}\left(x, \zeta^{\prime}\right)\left(i=1, \ldots, l_{1}\right)$ and $\mu_{j}\left(x, \zeta^{\prime}\right)\left(j=1, \ldots, l_{2}\right)$ are holomorphic for all $x, \zeta^{\prime}$ in a neighborhood of $x=0, \zeta^{\prime}=(1,0, \ldots, 0)$. Moreover, writing $\lambda_{i}(0 ; 1,0, \ldots, 0)=\lambda_{i}$ and $\mu_{j}(0 ; 1,0, \ldots, 0)=\mu_{j}$, we assume that $\lambda_{1}$, $\ldots, \lambda_{l_{1}}, \mu_{1}, \ldots, \mu_{l_{2}}$ are distinct.

Next, we impose condition (B) on the lower order terms. 
Condition (B). For each $j\left(j=1, \ldots, l_{1}\right)$ and for all $x, \zeta^{\prime}$ in $a$ neighborhood of $x=0, \zeta^{\prime}=(1,0, \ldots, 0)$, it holds that

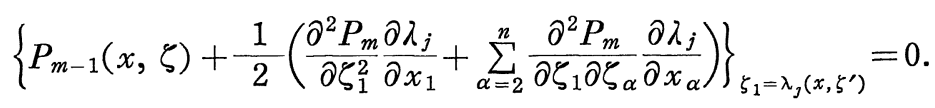

Now we can replace the above condition (A), (B) by the equivalent condition (C) defined by

Condition $(\mathbf{C})$. The differential operator $a\left(x, \frac{\partial}{\partial x}\right)$ is expressed in the form:

$$
\begin{aligned}
a\left(x, \frac{\partial}{\partial x}\right)= & h_{2}\left(x, \frac{\partial}{\partial x}\right)\left[h_{1}\left(x, \frac{\partial}{\partial x}\right)\right]^{2}+b\left(x, \frac{\partial}{\partial x}\right) h_{1}\left(x, \frac{\partial}{\partial x}\right) \\
& +c\left(x, \frac{\partial}{\partial x}\right),
\end{aligned}
$$

where $h_{1}(x, \zeta), h_{2}(x, \zeta)$ are the homogeneous polynomials of degree $l_{1}, l_{2}$ in $\zeta$, whose coefficients are holomorphic in a neighborhood of $x=0$. Moreover, the roots of the equations $h_{1}(0 ; \lambda, 1,0, \ldots, 0)=0, h_{2}(0 ; \mu, 1,0, \ldots, 0)=0$ are $\lambda_{i}\left(i=1, \ldots, l_{1}\right), \mu_{j}\left(j=1, \ldots, l_{2}\right) . \quad b(x, \zeta)$ and $c(x, \zeta)$ are any polynomials of degree $l_{1}+l_{2}-1$ and at most $m-2$ in $\zeta$, respectively.

In this paper, we use condition (C) instead of (A), (B) for the convenience of the calculus.

Now, as was shown in [2], we can construct the characteristic surfaces $K_{i}^{(1)}\left(i=1, \ldots, l_{1}\right), K_{j}^{(2)}\left(j=1, \ldots, l_{2}\right)$ for $h_{1}\left(x, \frac{\partial}{\partial x}\right), h_{2}\left(x, \frac{\partial}{\partial x}\right)$ issuing from $x_{1}=x_{2}=0$. Let $K_{i}^{(1)}$ and $K_{j}^{(2)}$ be expressed by the equations $\varphi^{(i)}(x)=0$ and $\psi^{(j)}(x)=0$ where

$$
\varphi^{(i)}\left(0, x^{\prime}\right)=x_{2}, \quad \phi^{(j)}\left(0, x^{\prime}\right)=x_{2}, \frac{\partial \varphi^{(i)}}{\partial x_{1}}(0)=\lambda_{i} \quad \text { and } \quad \frac{\partial \psi^{(j)}}{\partial x_{1}}(0)=\mu_{j} .
$$

The aggregation of $K_{i}^{(1)}\left(i=1, \ldots, l_{1}\right)$ and $K_{j}^{(2)}\left(j=1, \ldots, l_{2}\right)$ constitutes the characteristic surfaces for $P_{m}\left(x, \frac{\partial}{\partial x}\right)$ issuing from $x_{1}=x_{2}=0$.

Then our assertion may be stated as follows

Theorem. Assume that the differential operator $a\left(x, \frac{\partial}{\partial x}\right)$ satisfies 
condition (C). Then the solution $u(x)$ of the Cauchy problem (1.1) is analytic outside of $K_{1}^{(1)} \cup \cdots \cup K_{l_{1}}^{(1)} \cup K_{1}^{(2)} \cup \cdots \cup K_{l_{2}}^{(2)}$ and generally has singularities on $K_{1}^{(1)} \cup \cdots \cup K_{l_{1}}^{(1)} \cup K_{1}^{(2)} \cup \cdots \cup K_{l_{2}}^{(2)}$. More precisely, provided that the initial data have at most poles on $x_{2}=0, u(x)$ is expressed in the form

$$
\begin{aligned}
u(x)= & \sum_{i=1}^{l_{1}}\left\{\frac{A^{(i)}(x)}{\left[\varphi^{(i)}(x)\right]^{p_{\iota}}}+B^{(i)}(x) \log \varphi^{(i)}(x)\right\} \\
& +\sum_{j=1}^{l_{2}}\left\{\frac{C^{(j)}(x)}{\left[\psi^{(j)}(x)\right]^{q_{j}}}+D^{(j)}(x) \log \psi^{(j)}(x)\right\}+H(x),
\end{aligned}
$$

where $A^{(i)}(x), B^{(i)}(x), C^{(j)}(x), D^{(j)}(x)$ and $H(x)$ are holomorphic in a neighborhood of $x=0$ and $p_{i}, q_{j}$ are non-negative integers.

If at least one of $w_{k}\left(x^{\prime}\right)(k=0,1, \ldots, m-1)$ has essential singularity, $u(x)$ is expressed in the form

$$
\begin{aligned}
u(x)= & \sum_{i=1}^{l_{1}}\left\{\sum_{k=1}^{\infty} \frac{A_{k}^{(i)}(x)}{\left[\varphi^{(i)}(x)\right]^{k}}+B^{(i)}(x) \log \varphi^{(i)}(x)\right\} \\
& +\sum_{j=1}^{l_{2}}\left\{\sum_{k=1}^{\infty} \frac{C_{k}^{(j)}(x)}{\left[\psi^{(j)}(x)\right]^{k}}+D^{(j)}(x) \log \psi^{(j)}(x)\right\}+H(x),
\end{aligned}
$$

where $A_{k}^{(i)}(x), B^{(i)}(x), C_{k}^{(j)}(x)$ and $D^{(j)}(x)$ are holomorphic in a neighborhood of $x=0$.

Remark 1. As we see in the next section, when the initial conditions are given by

$$
\begin{aligned}
& \frac{\partial^{h} u}{\partial x_{1}^{h}}\left(0, x^{\prime}\right)=\frac{w\left(x^{\prime \prime}\right)}{x_{2}^{p}} \quad(p: \text { integer }>0) \\
& \frac{\partial^{k} u}{\partial x_{1}^{k}}\left(0, x^{\prime}\right)=0 \quad k \neq h \quad 0 \leqslant k \leqslant m-1,
\end{aligned}
$$

for the exponents $p_{i}\left(i=1, \ldots, l_{1}\right), q_{j}\left(j=1, \ldots, l_{2}\right)$ we have $p_{i} \leqslant p-h+1$ $\left(i=1, \ldots, l_{1}\right), q_{j} \leqslant p-h\left(j=1, \ldots, l_{2}\right)$.

Remark 2. The solution $u(x)$ has not always singularities on all the surfaces $K_{i}^{(1)}\left(i=1, \ldots, l_{1}\right), K_{j}^{(2)}\left(j=1, \ldots, l_{2}\right)$. There may occur that $u(x)$ 
is analytic on some $K_{i}^{(1)}, K_{j}^{(2)}$.

Remark 3. Condition (C) is a generalization of the condition by A. Lax [3] in the two dimensional space. A. Lax's condition in complex domain means that

$$
a\left(x, y ; \frac{\partial}{\partial x}, \frac{\partial}{\partial y}\right) u=\partial_{1}^{l_{1}} \ldots \partial_{s}^{l_{s}} u+\sum_{\substack{\nu_{1}+\ldots+\nu_{s} \leqslant l-1 \\ \nu_{1} \leqslant l_{1}, \ldots, \nu_{s} \leqslant l_{s}}} a_{\nu_{1}, \ldots, \nu_{s}}(x, y) \partial_{1}^{\nu_{1}} \ldots \partial_{s}^{\nu_{s}}
$$

where $\partial_{i}=\frac{\partial}{\partial x}+\lambda_{i}(x, y) \frac{\partial}{\partial y}, \lambda_{i}(x, y)(i=1, \ldots, s), a_{\nu_{1}, \ldots, \nu_{s}}(x, y)$ are holomorphic in a neighborhood of $(x, y)=(0,0)$ and $\lambda_{1}(0,0), \ldots, \lambda_{s}(0,0)$ are distinct.

Remark 4. In $C^{3}$, we consider the differential operator $a\left(x, \frac{\partial}{\partial x}\right)$ $=\frac{\partial^{2}}{\partial x_{1}^{2}}-\frac{\partial^{2}}{\partial x_{2} \partial x_{3}}$ with characteristic polynomial $\zeta_{1}^{2}-\zeta_{2} \zeta_{3}$. The equation $\zeta_{1}^{2}-\zeta_{2} \zeta_{3}=0$ has double root $\zeta_{1}=0$ for $\left(\zeta_{2}, \zeta_{3}\right)=(1,0)$, but this operator does not satisfy condition (A).

We shall give a remark on this operator in Section 4.

Remark 5. From the theorem it follows that the solution $u(x)$ is either ramified logarithmically or never ramified around each of $K_{i}^{(1)}$ $\left(i=1,2, \ldots, l_{1}\right)$ and $K_{j}^{(2)}\left(j=1, \ldots, l_{2}\right)$. However, unless operator $a\left(x, \frac{\partial}{\partial x}\right)$ satisfies condition (C), as we see in the following example where the characteristic surfaces touch each other, a solution can be found that is ramified finite times around a characteristic surface. The Cauchy problem

$$
\left\{\begin{array}{c}
\frac{\partial^{2} u}{\partial x^{2}}+2 x \frac{\partial^{2} u}{\partial x \partial y}=0 \\
u(0, y)=0 \\
\frac{\partial u}{\partial x}(0, y)=\frac{1}{y}
\end{array}\right.
$$

has the solution $u(x, y)$ in the form

$$
u(x, y)=\frac{1}{2 \sqrt{y}} \log \left(\frac{\sqrt{y}+x}{\sqrt{y}-x}\right)
$$


which is ramified two times around $y=0$.

Remark 6. We also remark the following:

Let $h_{1}(x, \zeta), h_{2}(x, \zeta)$ be the homogeneous polynomials of degree $l_{1}, l_{2}$ in $\zeta$ with holomorphic coefficients, and $b(x, \zeta), c(x, \zeta)$ be any polynomials of degree $l_{1}+l_{2}-1$, at most $m-2$ in $\zeta$ with holomorphic coefficients.

Let $S$ be a regular surface defined by $\varphi(x)=0\left(\left(\varphi_{x}\right) \neq 0\right)$ which is simple characteristic for $h_{1}\left(x, \frac{\partial}{\partial x}\right)$, but non-characteristic for $h_{2}\left(x, \frac{\partial}{\partial x}\right)$.

Now we consider the differential equation

$$
a\left(x, \frac{\partial}{\partial x}\right) u(x)=0
$$

where

$$
\begin{aligned}
a\left(x, \frac{\partial}{\partial x}\right)= & h_{2}\left(x, \frac{\partial}{\partial x}\right)\left[h_{1}\left(x, \frac{\partial}{\partial x}\right)\right]^{2}+b\left(x, \frac{\partial}{\partial x}\right) h_{1}\left(x, \frac{\partial}{\partial x}\right) \\
& +c\left(x, \frac{\partial}{\partial x}\right) .
\end{aligned}
$$

Then, we have the solution of this equation with singularity on $S$ in the form

$$
\begin{array}{rlrl}
u(x) & =[\varphi(x)]^{s} H_{1}(x)+H_{2}(x) & & \text { for } \quad s \neq-1,-2, \ldots \\
& =[\varphi(x)]^{s} H_{1}(x)+H_{2}(x) \log \varphi(x)+H_{3}(x) & \text { for } \quad s=-1,-2, \ldots,
\end{array}
$$

where $H_{1}(x), H_{2}(x)$ and $H_{3}(x)$ are holomorphic in a neighborhood of $x=0$ and $H_{1}(0) \neq 0$.

In the next section we shall construct the formal solution of our problem. Section 3 is devoted to the proof of its exactness. In Section 4 we shall give a remark on the existence domain of the solution.

\section{$\S 2$. Construction of Formal Solution}

Let $a\left(x, \frac{\partial}{\partial x}\right)$ be a linear differential operator satisfying condition (C). Our aim of this section is to construct the formal solution of the Cauchy problem (1.1) in the case where the initial data have at most poles. The principle of superposition allows us to reduce our problem to the 
special case as follows

$$
a\left(x, \frac{\partial}{\partial x}\right) u(x)=0
$$

with the initial conditions

$$
\left\{\begin{array}{l}
\frac{\partial^{k} u}{\partial x_{1}^{k}}\left(0, x^{\prime}\right)=0 \quad k \neq h, \quad 0 \leqslant k \leqslant m-1 \\
\frac{\partial^{h} u}{\partial x_{1}^{h}}\left(0, x^{\prime}\right)=(-1)^{p}(p-1) ! \frac{w\left(x^{\prime \prime}\right)}{x_{2}^{p}},
\end{array}\right.
$$

where $p$ is integer $>0$ and $w\left(x^{\prime \prime}\right)$ is holomorphic function of $x^{\prime \prime}=\left(x_{3}, \ldots, x_{n}\right)$ in a neighborhood of $x^{\prime \prime}=0$.

Now, in order to construct the formal solution of (2.1) and (2.2), we introduce the auxiliary functions $f_{j}(s)(j=-m,-m+1, \ldots \ldots)$ satisfying the following relations:

$$
\left\{\begin{array}{rlrl}
\frac{d}{d s} f_{j}(s) & =f_{j-1}(s) & & \\
f_{0}(s) & =\log s & f_{1}(s)=s \log s-s \\
f_{-1}(s) & =\frac{1}{s} & & f_{\alpha}(s)=\frac{s^{\alpha}}{\alpha !} \log s-\frac{A_{\alpha}}{\alpha !} s^{\alpha} \\
\vdots & A_{\alpha}=1+\frac{1}{2}+\cdots+\frac{1}{\alpha} \\
f_{-m}(s)=(-1)^{m-1}(m-1) ! \frac{1}{s^{m}} & & \alpha: \text { integer }>0 .
\end{array}\right.
$$

In terms of these functions, we expand the formal solution $u(x)$ of (2.1) and (2.2) in the form

$$
u(x)=\sum_{i=1}^{l_{1}} \sum_{k=-p+h-1}^{\infty} f_{k}\left(\varphi^{(i)}\right) u_{k}^{(i)}+\sum_{j=1}^{l_{2}} \sum_{k=-p+h}^{\infty} f_{k}\left(\psi^{(j)}\right) v_{k}^{(j)} .
$$

In the following we shall determine these coefficients $u_{k}^{(i)}, v_{k}^{(j)}$. In order to do so, we shall repeatedly use Leibniz' formula

$$
h\left(x, \frac{\partial}{\partial x}\right) f(\varphi) u=f^{(m)}(\varphi) h\left(x, \varphi_{x}\right) u
$$




$$
+f^{(m-1)}(\varphi)\left[\sum_{i=1}^{n} h^{(i)}\left(x, \varphi_{x}\right) \frac{\partial u}{\partial x_{i}}+c(x) u\right]+\sum_{\alpha=2}^{m} f^{(m-\alpha)}(\varphi) L_{\alpha}[u]
$$

where $L_{\alpha}(\alpha=2, \ldots, m)$ are differential operators of order $\alpha$ depending only on the differential operator $h\left(x, \frac{\partial}{\partial x}\right)$.

Now, substituting (2.4) into (2.1) and setting the coefficients of $f_{k-m+2}\left(\varphi^{(i)}\right)$ and $f_{k-m+1}\left(\psi^{(j)}\right)(k=-p+h-1,-p+h, \ldots)$ equal to zero, we obtain the recursion formulas for $u_{k}^{(i)}$ and $v_{k}^{(j)}$

$$
\begin{aligned}
& \widetilde{L_{0}} L_{1} L_{1}\left[u_{k}^{(i)}\right]+\underset{\widetilde{L}_{0}}{\approx} L_{1}\left[u_{k}^{(i)}\right]+\underset{\widetilde{L}_{0}}{\left.\widetilde{\approx}_{k}^{(i)}\right]} \\
& =\sum_{\alpha=0}^{m-3} \mathscr{L}_{\alpha+3}\left[u_{k-1-\alpha}^{(i)}\right]
\end{aligned}
$$

and

$$
\widetilde{L_{1}} L_{0} L_{0}\left[v_{k}^{(j)}\right]+\underset{\widetilde{L}_{0}}{\approx} L_{0}\left[v_{k}^{(j)}\right]=\sum_{\alpha=0}^{m-2} \mathscr{L}_{\alpha+2}\left[v_{k-1-\alpha}^{(j)}\right]
$$

where

$$
\left\{\begin{array}{l}
L_{0}=h_{1}\left(x, \phi_{x}^{(j)}\right) \\
\widetilde{L}_{0}=h_{2}\left(x, \varphi_{x}^{(i)}\right) \\
\widetilde{L}_{0}=b_{l_{1}+l_{2}-1}\left(x, \varphi_{x}^{(i)}\right) \\
\widetilde{\widetilde{L}}_{0}=b_{l_{1}+l_{2}-1}\left(x, \psi_{x}^{(j)}\right) \quad \mathbb{\widetilde { L }}_{0}=c_{m-2}\left(x, \varphi_{x}^{(i)}\right) \\
L_{1}=\sum_{\alpha=1}^{n} h_{1}^{(\alpha)}\left(x, \varphi_{x}^{(i)}\right) \frac{\partial}{\partial x_{\alpha}}+c(x) \\
\widetilde{L}_{1}=\sum_{\alpha=1}^{n} h_{2}^{(\alpha)}\left(x, \psi_{x}^{(j)}\right) \frac{\partial}{\partial x_{\alpha}}+c^{\prime}(x) \\
\mathscr{L}_{\alpha} \text { are differential operators of order } \alpha .
\end{array}\right.
$$

We note that, from our hypothesis (C), $h_{1}\left(0 ; \mu_{j}, 1,0, \ldots, 0\right) \neq 0$ $\left(j=1, \ldots, l_{2}\right), h_{2}\left(0 ; \lambda_{i}, 1,0, \ldots, 0\right) \neq 0\left(i=1, \ldots, l_{1}\right)$, and hence $L_{0}$ and $\widetilde{L}_{0}$ do not vanish in a neighborhood of $x=0$.

Now, we wish to rewrite (2.6) in a simpler form. In order to do 
so, we introduce the well-known ordinary differential equation

$$
\left\{\begin{array}{c}
\frac{d x_{1}}{d t}=h_{1}^{(1)}\left(x, \varphi_{x}^{(i)}\right) \\
\vdots \\
\frac{d x_{n}}{d t}=h_{1}^{(n)}\left(x, \varphi_{x}^{(i)}\right)
\end{array}\right.
$$

with initial data $x_{1}(0)=0, x_{k}(0)=y_{k}(k=2, \ldots, n)$. Let $x_{j}=\xi_{j}^{(i)}(t, y)$ $(j=1, \ldots, n)$ be the solution of this system, which defines the bicharacteristic curve for $h_{1}\left(x, \frac{\partial}{\partial x}\right)$ and generates $K_{i}^{(1)}$. By composing $u_{k}^{(i)}(x)$ and $x_{j}=\xi_{j}^{(i)}(t, y)(j=1,2, \ldots, n)$, we define $U_{k}^{(i)}(t, y)$ :

$$
U_{k}^{(i)}(t, y)=u_{k}^{(i)}\left(\xi_{1}^{(i)}(t, y), \ldots, \xi_{n}^{(i)}(t, y)\right) \quad y=\left(y_{2}, \ldots, y_{n}\right) .
$$

Then, evidently we have

$$
\frac{\partial U_{k}^{(i)}}{\partial t}=L_{1}\left[u_{k}^{(i)}\right]
$$

Therefore, we can rewrite (2.6) with $k$ replaced by $-p+h-1+k$ as follows :

$$
\begin{aligned}
& {\left[\left(\frac{\partial}{\partial t}\right)^{2}+a_{i}(t, y) \frac{\partial}{\partial t}+b_{i}(t, y)\right] U_{-p+h-1+k}^{(i)}} \\
& \quad=\sum_{\alpha=0}^{m-3} \mathscr{L}_{\alpha+3}\left[U_{-p+h-2+k-\alpha}^{(i)}\right],
\end{aligned}
$$

where $\mathscr{L}_{\alpha}$ are differential operators of order $\alpha$ with holomorphic coefficients and $a_{i}(t, y), b_{i}(t, y)$ are holomorphic in a neighborhood of $(t, y)=(0,0)$. In particular,

$$
\left[\left(\frac{\partial}{\partial t}\right)^{2}+a_{i}(t, y) \frac{\partial}{\partial t}+b_{i}(t, y)\right] U_{-p+h-1}^{(i)}=0
$$

Thus, we see that $u_{k}^{(i)}$ satisfies the second order differential equation along bicharacteristic curve.

Next, we perform the same procedure for (2.7) as (2.6). Namely, we consider the ordinary differential equation 


$$
\left\{\begin{aligned}
\frac{d x_{1}}{d t} & =h_{2}^{(1)}\left(x, \psi_{x}^{(i)}\right) \\
\vdots & \\
\frac{d x_{n}}{d t} & =h_{2}^{(n)}\left(x, \psi_{x}^{(i)}\right)
\end{aligned}\right.
$$

with the initial data $x_{1}(0)=0, x_{k}(0)=y_{k}(k=2, \ldots, n)$. Let $\eta_{j}^{(i)}(t, y)$ $(j=1,2, \ldots, n)$ be the solution of this system and put

$$
V_{k}^{(j)}(t, y)=v_{k}^{(j)}\left(\eta_{1}^{(j)}(t, y), \ldots, \eta_{n}^{(j)}(t, y)\right) .
$$

Then, we can rewrite (2.7) as follows

$$
\left[\frac{\partial}{\partial t}+c_{j}(t, y)\right] V_{-p+h+k}^{(j)}(t, y)=\sum_{\alpha=0}^{m-2} \mathscr{L}_{\alpha+2}\left[V_{-p+h+k-1-\alpha}^{(j)}\right],
$$

where $\mathscr{L}_{\alpha}$ are differential operators of order $\alpha$ and $c_{j}(t, y)$ is holomorphic in a neighborhood of $(t, y)=(0,0)$. In particular,

$$
\left[\frac{\partial}{\partial t}+c_{j}(t, y)\right] V_{-p+h}^{(j)}(t, y)=0
$$

In order to seek successively $U_{-p+h-1+k}^{(i)}$ and $V_{-p+h+k}^{(j)}$, we must determine the initial data $U_{-p+h-1+k}^{(i)}(0, y), \frac{\partial U_{-p+h-1+k}^{(i)}}{\partial t}(0, y)$ and $V_{-p+h{ }_{k}}^{(j)}(0, y)$. This is done by a consideration of the initial data (2.2). Indeed, we have for $0 \leqslant \alpha \leqslant m-1$ the identity

$$
\begin{aligned}
\left\{\left.\frac{\partial^{\alpha} u}{\partial x_{1}^{\alpha}}\right|_{x_{1}=0}=\right. & \left\{\sum _ { i = 1 } ^ { l _ { 1 } } \sum _ { k = - p + h - 1 } ^ { \infty } \left(f_{k-\alpha}\left(\varphi^{(i)}\right)\left(\frac{\partial \varphi^{(i)}}{\partial x_{1}}\right)^{\alpha} u_{k}^{(i)}\right.\right. \\
& +f_{k-\alpha+1}\left(\varphi^{(i)}\right)\left[\alpha\left(\frac{\partial \varphi^{(i)}}{\partial x_{1}}\right)^{\alpha-1} \frac{\partial u_{k-1}^{(i)}}{\partial x_{1}}+c(x) u_{k-1}^{(i)}\right] \\
& \left.\left.+\sum_{\beta=2}^{m-1} f_{k-\alpha+\beta}\left(\varphi^{(i)}\right) \mathfrak{M}_{\beta}\left[u_{k-\beta}^{(i)}\right]\right)\right\}_{x_{1}=0} \\
& +\left\{\sum _ { j = 1 } ^ { l _ { 2 } } \sum _ { k = - p + h } ^ { \infty } \left(f_{k-\alpha}\left(\psi^{(j)}\right)\left(\frac{\partial \psi^{(j)}}{\partial x_{1}}\right)^{\alpha} v_{k}^{(j)}\right.\right. \\
& +f_{k-\alpha+1}\left(\psi^{(i)}\right)\left[\alpha\left(\frac{\partial \psi^{(j)}}{\partial x_{1}}\right)^{\alpha-1} \frac{\partial v_{k-1}^{(j)}}{\partial x_{1}}+c(x) v_{k-1}^{(j)}\right] \\
& \left.\left.+\sum_{\beta=2}^{m-1} f_{k-\alpha+\beta}\left(\psi^{(j)}\right) \mathfrak{M}_{\beta}\left[v_{k-\beta}^{(j)}\right]\right)\right\}_{x_{1}=0},
\end{aligned}
$$


where we have denoted by the same notation $\mathfrak{M}_{\alpha}(\alpha=1,2, \ldots, m-1)$ the various differential operators of order $\alpha$. We note that these operators depend only on the differential operator $a\left(x, \frac{\partial}{\partial x}\right)$ and so of course are independent of $k$. By substituting this into (2.2) and setting the coefficients of $f_{j}\left(x_{2}\right)(j=-p+h-m, \ldots \ldots)$ equal to zero, we obtain the simultaneous linear equation for $u_{-p+h+k}^{(i)}\left(0, x^{\prime}\right), \frac{\partial u_{-p+h+k-1}^{(i)}}{\partial x_{1}}\left(0, x^{\prime}\right)$ and $v_{-p+h+k}^{(j)}\left(0, x^{\prime}\right)$. The determinant that consists of the coefficients of these unknowns is

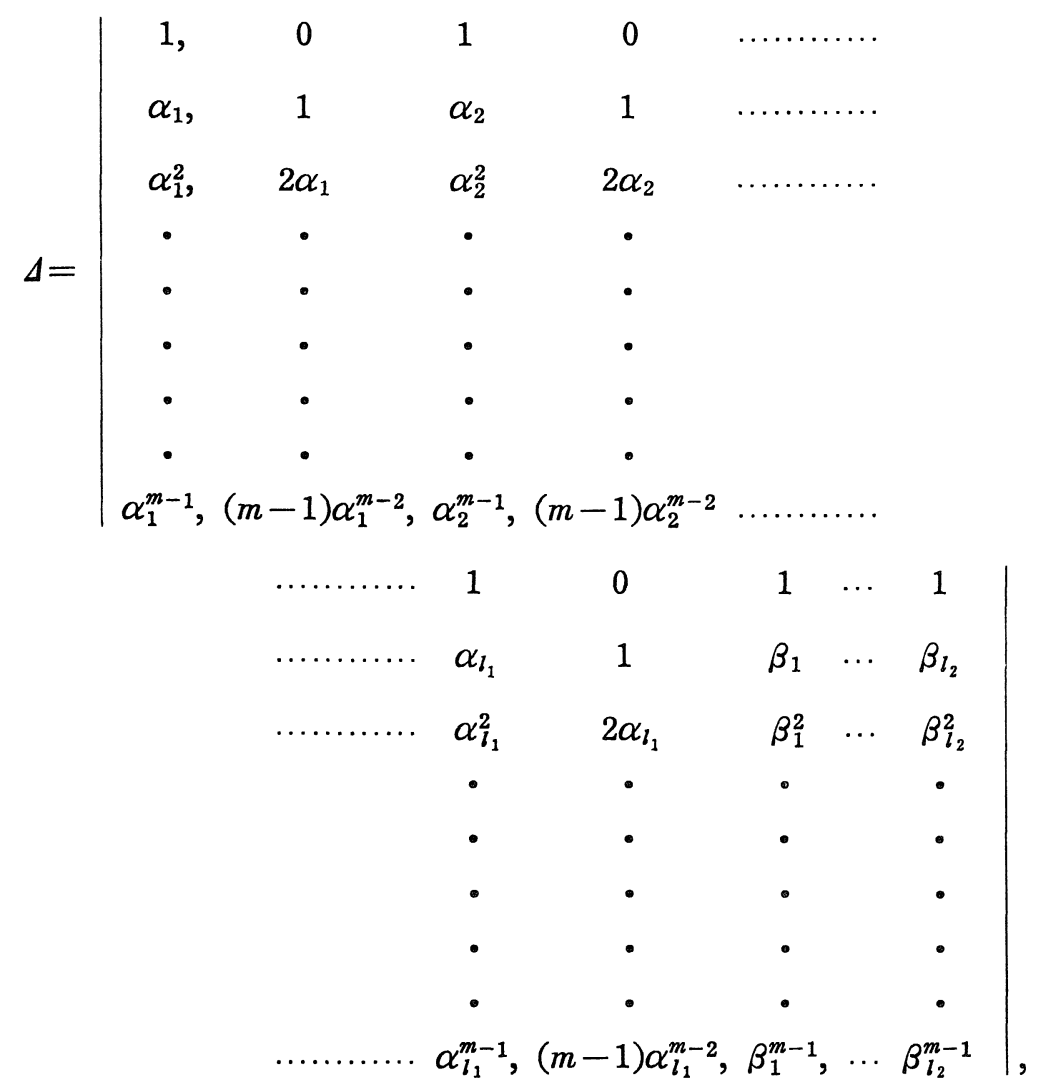

where

$$
\alpha_{i}\left(x^{\prime}\right)=\frac{\partial \varphi^{(i)}}{\partial x_{1}}\left(0, x^{\prime}\right)\left(i=1, \ldots, l_{1}\right) \text { and } \beta_{j}\left(x^{\prime}\right)=\frac{\partial \psi^{(j)}}{\partial x_{1}}\left(0, x^{\prime}\right)\left(j=1, \ldots, l_{2}\right) .
$$

Now, from the fact that $\alpha_{i}(0)=\lambda_{i}\left(i=1, \ldots, l_{1}\right), \beta_{j}(0)=\mu_{j}\left(j=1, \ldots, l_{2}\right)$, 
these functions $\alpha_{1}\left(x^{\prime}\right), \ldots, \alpha_{l_{1}}\left(x^{\prime}\right), \beta_{1}\left(x^{\prime}\right), \ldots, \beta_{l_{2}}\left(x^{\prime}\right)$ are distinct in a neighborhood of $x^{\prime}=0$. Therefore this determinant $\Delta$ does not vanish in a neighborhood of $x^{\prime}=0$. Hence, we get

$$
u_{-p+h-1}^{(i)}\left(0, x^{\prime}\right)=0 \quad i=1, \ldots, l_{1} \text {. }
$$

and

$$
\begin{aligned}
& u_{-p+h}^{(i)}\left(0, x^{\prime}\right)=\Delta_{1}\left(h, i, x^{\prime}\right) w\left(x^{\prime \prime}\right) \\
& v_{-p+h}^{(j)}\left(0, x^{\prime}\right)=\Delta_{2}\left(h, j, x^{\prime}\right) w\left(x^{\prime \prime}\right) \\
& \frac{\partial u_{-p+h-1}^{(i)}}{\partial x_{1}}\left(0, x^{\prime}\right)=\Delta_{3}\left(h, i, x^{\prime}\right) w\left(x^{\prime \prime}\right),
\end{aligned}
$$

where $\Delta_{1}, \Delta_{2}, \Delta_{3}$ are holomorphic in a neighborhood of $x^{\prime}=0$ and depend only on $a\left(x, \frac{\partial}{\partial x}\right), h$ and $i, j . \quad$ For general $k \geqq 1$, we can express $u_{-p+h+k}^{(i)}, v_{-p+h+k}^{(j)}, \frac{\partial u_{-p+h+k-1}^{(i)}}{\partial x_{1}}$ respectively in the form

$$
\left.\begin{array}{c}
u_{-p+h+k}^{(i)}\left(0, x^{\prime}\right) \\
v_{-p+h+k}^{(j)}\left(0, x^{\prime}\right) \\
\frac{\partial u_{-p+h+k-1}^{(i)}}{\partial x_{1}}\left(0, x^{\prime}\right)
\end{array}\right\}=\left\{\begin{array}{l}
\sum_{\alpha=1}^{l_{1}} \mathfrak{M}_{0}^{(\alpha)}\left[u_{-p+h+k-1}^{(\alpha)}\right] \\
+\sum_{\alpha=1}^{l_{1}} \sum_{p_{\alpha}=2}^{m-1} \mathfrak{M}_{p_{\alpha}}^{(\alpha)}\left[u_{-p+h+k-p_{\alpha}}^{(\alpha)}\right] \\
+\sum_{\alpha=1}^{l_{2}} \sum_{p_{\alpha}=1}^{m-1} \mathfrak{M}_{p_{\alpha}}^{(\alpha)}\left[v_{-p+h+k-p_{\alpha}}^{(\alpha)}\right] .
\end{array}\right.
$$

Now, we wish to transform (2.16), (2.17), (2.18) into the expressions for $U_{-p+h+k}^{(i)}(0, y), \frac{\partial U_{-p+h+k-1}^{(i)}}{\partial t}(0, y), V_{-p+h+k}^{(j)}(0, y)$. At first, obviously (2.16), (2.17) imply that

$$
\begin{aligned}
& U_{-p+h-1}^{(i)}(0, y)=0 \\
& U_{-p+h}^{(i)}(0, y)=\Delta_{1}(h, i, y) w\left(y^{\prime}\right) . \quad y^{\prime}=\left(y_{3}, \ldots, y_{n}\right) \\
& V_{-p+h}^{(j)}(0, y)=\Delta_{2}(h, j, y) w\left(y^{\prime}\right) .
\end{aligned}
$$

Taking account of (2.16) and (2.17), we also see that

$$
\frac{\partial U_{-p+h-1}^{(i)}}{\partial t}(0, y)=h_{1}^{(1)}\left(x, \varphi_{x}^{(i)}\right) \frac{\partial u_{-p+h-1}^{(i)}}{\partial x_{1}}
$$




$$
\begin{gathered}
+\left.\sum_{\alpha=2}^{n} h_{1}^{(\alpha)}\left(x, \varphi_{x}^{(i)}\right) \frac{\partial u_{-p+h-1}^{(i)}}{\partial x_{\alpha}}\right|_{\begin{array}{l}
x_{1}=0 \\
x^{\prime}=y
\end{array}} \\
=\left.h_{1}^{(1)}\left(x, \varphi_{x}^{(i)}\right) \Delta_{3}\left(h, i, x^{\prime}\right) w\left(x^{\prime \prime}\right)\right|_{\begin{array}{l}
x_{1}=0 \\
x^{\prime}=y
\end{array}} .
\end{gathered}
$$

Next, we pass to $\frac{\partial U_{-p+h+k-1}^{(i)}}{\partial t}(0, y)(k \geqq 1)$. We have

$$
\begin{aligned}
\left.\frac{\partial U_{-p+h+k-1}^{(i)}}{\partial t}\right|_{t=0}= & h_{1}^{(1)}\left(x, \varphi_{x}^{(i)}\right) \frac{\partial u_{-p+h+k-1}^{(i)}}{\partial x_{1}} \\
& +\left.\sum_{\alpha=2}^{n} h_{1}^{(\alpha)}\left(x, \varphi_{x}^{(i)}\right) \frac{\partial u_{-p+h+k-1}^{(i)}}{\partial x_{\alpha}}\right|_{\substack{x_{1}=0 \\
x^{\prime}=y}}
\end{aligned}
$$

and so, combining this with (2.18), we find the following expression

$$
\begin{aligned}
\frac{\partial U_{-p+h+k-1}^{(i)}}{\partial t}(0, y)= & \sum_{j=1}^{l_{1}} \mathfrak{M}_{0}^{(j, i)}\left[U_{-p+h+k-1}^{(j)}\right] \\
& +\sum_{j=1}^{l_{1}} \sum_{p_{j}=2}^{m} \mathfrak{M}_{p_{j}}^{(j, i)}\left[U_{-p+h+k-p_{j}}^{(j)}\right] \\
& +\sum_{j=1}^{l_{2}} \sum_{p_{j}=1}^{m} \mathfrak{M}_{p_{j}}^{(j, i)}\left[V_{-p+h+k-p_{j}}^{(j)}\right],
\end{aligned}
$$

where $\mathfrak{M}_{\alpha}$ are differential operators of order $\alpha$ which consists of derivatives with respect to $t$ and $y$, and depend only on $a\left(x, \frac{\partial}{\partial x}\right)$. Thus, we obtain the expressions for $U_{-p+h+k}^{(i)}(0, y), V_{-p+h+k}^{(j)}(0, y)$ and $\frac{\partial U_{-p+h+k-1}^{(i)}}{\partial t}(0, y)$ in the form

$$
\begin{array}{r}
\left.\left.\begin{array}{c}
U_{-p+h+k}^{(i)}(0, y) \\
V_{-p+h+k}^{(j)}(0, y)
\end{array}\right\}=\begin{array}{l}
\left\{\sum_{j=1}^{l_{1}} \mathfrak{M}_{0}^{(j, i)}\left[U_{-p+h+k-1}^{(j)}\right]\right. \\
\frac{\partial U_{-p+h+k-1}^{(i)}}{\partial t}(0, y)
\end{array}\right\} \sum_{j=1}^{l_{1}} \sum_{p_{j}=2}^{m} \mathfrak{M}_{p_{j}}^{(j, i)}\left[U_{-p+h+k-p_{j}}^{(j)}\right] \\
\\
\left.+\sum_{j=1}^{l_{2}} \sum_{p_{j}=1}^{m} \mathfrak{M}_{p_{j}}^{(j, i)}\left[V_{-p+h+k-p_{j}}^{(j)}\right]\right\}_{t=0} .
\end{array}
$$

Summing up the results that we have obtained in this section, we reach the following conclusion:

$U_{-p+h-1}^{(i)}(t, y)$ and $V_{-p+h}^{(j)}(t, y)$ are obtained by solving the second 
order ordinary differential equation (2.11) with the initial data (2.19), (2.22) and the first order ordinary differential equation (2.13) with the initial data (2.21) respectively. Furthermore, if we obtain $U_{-p+h-1+k}^{(i)}(t, y)$, $V_{-p+h+k}^{(j)}(t, y)$ for $k=0,1, \ldots, k-1$, we can determine $U_{-p+h-1+k}^{(i)}(t, y)$, $V_{-p+h+k}^{(j)}(t, y)$ by integrating the second order ordinary differential equation (2.10) with the initial data given by (2.24), the first order ordinary differential equation (2.12) with the initial data given by (2.24), respectively.

\section{$\S 3$. The Exactness of the Formal Solution}

In this section, we shall prove the exactness of the formal solution (2.4) constructed in the above section. This is done by the estimates for $u_{k}^{(i)}$ and $v_{k}^{(j)}$. Such estimates are obtained from Propositions due to Mizohata [6] and [7] (especially [7] §5).

Proposition 1. Let $a(x)$ and $b(x)$ be two holomorphic functions. We assume

$$
\begin{aligned}
& \left|D^{\nu} a(x)\right| \leqslant \frac{(r+|\nu|) !}{(k \rho)^{|\nu|}} A \quad(k>1) \\
& \left|D^{\nu} b(x)\right| \leqslant \frac{(s+|\nu|) !}{\rho^{|\nu|}} B
\end{aligned}
$$

where $r$ and $s$ are non negative integers. Then we have the following estimate:

$$
\left|D^{\nu}(a b)(x)\right| \leqslant \frac{(r+s+|\nu|) !}{\rho^{|\nu|}} \frac{k}{k-1} A B / C_{s}^{r+s} .
$$

We consider an ordinary differential operator with parameter

$$
\Pi=\left(\frac{\partial}{\partial t}\right)^{l}+\sum_{s=0}^{l-1} a_{s}(t, x)\left(\frac{\partial}{\partial t}\right)^{s},
$$

where the coefficients $a_{s}(t, x)$ are holomorphic in a neighborhood of $(0,0)$ and satisfy

$$
\left|D_{t, x}^{\nu} a_{s}(t, x)\right| \leqslant \frac{|\nu| !}{(3 \rho)^{|\nu|} \gamma}
$$

Then we have 
Proposition 2. Under the above condition, we consider the solution $u(t, x)$ of $\Pi[u]=f(t, x)$ with initial data $\left(D_{t}^{s} u\right)(0, x)=0,0 \leqslant s \leqslant l-1$. Assume that

$$
\begin{aligned}
& \left|D_{x}^{\nu} D_{t}^{q} f(t, x)\right| \\
& \quad \leqslant \frac{(r+q+|\nu|) !}{\rho^{q+|\nu|}} \exp (\operatorname{lr}|t|) K(|t|)^{r+q+|\nu|}(l \gamma)^{q} A .
\end{aligned}
$$

Then we have

$$
\begin{aligned}
& \left|D_{t}^{q} D_{x}^{\nu} u(t, x)\right| \\
& \quad \leqslant 2^{l} \frac{(r-l+q+|\nu|) !}{\rho^{q+|\nu|}} \exp (\operatorname{lr}|t|) K(|t|)^{r+q+|\nu|}(l \gamma)^{q} A,
\end{aligned}
$$

where $K(t)=\exp (l \gamma t)(1+l \gamma t), \gamma \geq 27,0<\rho \leqslant \frac{1}{18}$.

Proposition 3. Let $u(t, x)$ be the solution of the equation

$$
\Pi[u]=0
$$

with initial data

$$
\left\{\begin{array}{l}
\left|D_{x}^{\nu} u(0, x)\right| \leqslant \frac{(r-l+1+|\nu|) !}{\rho^{|\nu|}} A \\
\left|D_{x}^{\nu}\left(\frac{\dot{\partial}}{\partial t}\right)^{l-1} u(0, x)\right| \leqslant \frac{(r+|\nu|) !}{\rho^{|\nu|}} A .
\end{array}\right.
$$

Then we have

$$
\begin{aligned}
& \left|D_{t}^{q} D_{x}^{\nu} u(t, x)\right| \\
& \quad \leqslant 2^{l} \frac{(r-l+1+q+|\nu|) !}{\rho^{q+|\nu|}} \exp (l \gamma|t|) K(|t|)^{r+q+|\nu|}(l \gamma)^{q} A .
\end{aligned}
$$

Once these propositions have been established, we can give estimates for $U_{-p+h+k-1}^{(i)}$ and $V_{-p+h+k}^{(j)}$ by following lines of reasoning similar to that of [2].

Since the coefficients $a_{i}(t, y), b_{i}(t, y)$ and $c_{j}(t, y)$ in the equations (2.10) and (2.12) are holomorphic in a neighborhood of $(0,0)$, we can assume that 


$$
\left.\begin{array}{l}
\left|D_{t, y}^{\nu} a_{i}(t, y)\right| \\
\left|D_{t, y}^{\nu} b_{i}(t, y)\right| \\
\left|D_{t, y}^{\nu} c_{j}(t, y)\right|
\end{array}\right\} \leqslant \frac{|\nu| !}{(3 \rho)^{|\nu|} \gamma}
$$

with suitable constants $\gamma(\geqslant 27), \rho\left(<\frac{1}{18}\right)$.

Now, let us assume that $w\left(y^{\prime}\right)$ in (2.2) satisfies

$$
\left|D_{y}^{\nu} w\left(y^{\prime}\right)\right| \leqslant \frac{A}{\rho^{|\nu|}}(r+|\nu|) !
$$

where $A=\max _{\left|y^{\prime}\right| \leqslant \rho}\left|w\left(y^{\prime}\right)\right|$ and $r$ is integer $\geq 1$. Then we have for general $k \geq 0$

$\left(P_{k}\right)$

$$
\begin{aligned}
& \left|D_{t}^{q} D_{y}^{\nu} U_{-p+h-1+k}^{(i)}(t, y)\right| \\
& \leqslant A B^{k+1} \frac{(r+k-1+|\nu|+q) !}{\rho^{|\nu|+q}} \exp (2 \gamma|t|) K(|t|)^{r+k+|\nu|+q}(2 \gamma)^{q}
\end{aligned}
$$

$\left(Q_{k}\right)$

$$
\begin{aligned}
& \left|D_{t}^{q} D_{y}^{\nu} V_{-p+h+k}^{(j)}(t, y)\right| \\
& \leqslant A B^{k+1} \frac{(r+k+|\nu|+q) !}{\rho^{|\nu|+q}} \exp (\gamma|t|) K(|t|)^{r+k+|\nu|+q}(\gamma)^{q}
\end{aligned}
$$

and

$$
D_{y}^{\nu}\left\{\begin{array}{c}
U_{-p+h+k}^{(i)} \\
V_{-p+h+k}^{(j)} \\
\frac{\partial U_{-p+h+k-1}^{(i)}}{\partial t}
\end{array}\right\}(0, y) \mid \leqslant B^{k+1} A \frac{(r+k+|\nu|) !}{\rho^{|\nu|}}
$$

with some constant $B(>1)$ depending only on $a\left(x, \frac{\partial}{\partial x}\right)$.

We shall prove these estimates $\left(P_{k}\right),\left(Q_{k}\right)$ and $\left(I_{k}\right)$ by induction on $k$.

Let us start with $k=0$. Since $\Delta_{1}, \Delta_{2}, \Delta_{3}$ in (2.20), (2.21), (2.22) are holomorphic in a neighborhood of $y=0$, we can find a constant $E$ such that 


$$
\left\{\begin{array}{l}
\left|D_{y}^{\nu} \Delta_{1}\right| \\
\left|D_{y}^{\nu} \Delta_{2}\right| \\
\mid D_{y}^{\nu}\left\{h_{1}^{(1)}\left(x, \varphi_{x}^{(i)}\right) \Delta_{3}\right\} \begin{array}{c}
x_{1}=0 \mid \\
x^{\prime}=y
\end{array}
\end{array}\right\} \leqslant \frac{E}{(3 \rho)^{|\nu|}} .
$$

Hence, applying Proposition 1 to (2.20), (2.21) and (2.22), and taking account of (3.2) and (3.3), we get

$$
D_{y}^{\nu}\left\{\begin{array}{c}
U_{-p+h}^{(i)} \\
V_{-p+h}^{(j)} \\
\frac{\partial U_{-p+h-1}^{(i)}}{\partial t}
\end{array}\right\}(0, y) \mid \leqslant 2 A E \frac{(r+|\nu|) !}{\rho^{|\nu|}} .
$$

This and (2.19) lead to estimates for the initial data $U_{-p+h-1}^{(i)}(0, y)$ $\frac{\partial U_{-p+h-1}^{(i)}}{\partial t}(0, y)$ of the equation (2.11), and so, applying Proposition 3 with $l=2$ to (2.11), we get

$$
\begin{aligned}
& \left|D_{t}^{q} D_{y}^{\nu} U_{-p+h-1}(t, y)\right| \\
& \quad \leqslant 2^{3} E A \frac{(r-1+|\nu|+q) !}{\rho^{|\nu|+q}} \exp (2 \gamma|t|) K(|t|)^{r+q+|\nu|}(2 \gamma)^{q} .
\end{aligned}
$$

Quite similarly, since (3.4) contains the estimate for the initial data $V_{-p+h}^{(j)}(0, y)$ of the equation (2.13), Proposition 3 with $l=1$ yields that

$$
\begin{aligned}
& \left|D_{t}^{q} D_{y}^{\nu} V_{-p+h}^{(j)}(t, y)\right| \\
& \quad \leqslant 2^{2} E A \frac{(r+|\nu|+q) !}{\rho^{|\nu|+q}} \exp (\gamma|t|) K(|t|)^{r+q+|\nu|}(\gamma)^{q} .
\end{aligned}
$$

Consequently, if we take for $B$ a constant so that $B \geq 2^{3} E$, from (3.4), (3.5) and (3.6) we see that $\left(P_{0}\right),\left(Q_{0}\right)$ and $\left(I_{0}\right)$ are valid. Now, we assume that $\left(P_{k}\right),\left(Q_{k}\right)$ and $\left(I_{k}\right)$ have already been proved for $k=0,1, \ldots, k$. We wish to assert that $\left(P_{k+1}\right),\left(Q_{k+1}\right)$ and $\left(I_{k+1}\right)$ are also valid.

We first consider $\left(I_{k+1}\right)$. We recall that $U_{-p+h+k+1}^{(i)}(0, y)$, $V_{-p+h+k+1}^{(j)}(0, y)$ and $\frac{\partial U_{-p+h+k}^{(i)}}{\partial t}(0, y)$ are expressed in the similar form (2.24). Let us apply the inductive hypothesis and Proposition 1 to the terms in the right hand side of (2.24) with $k$ replaced by $k+1$, then we 
get

$\mid D_{y}^{\nu}$ the right hand side of (2.24) with $k$ replaced by $\left.k+1\right\} \mid$

$$
\begin{aligned}
\leqslant & A B^{k+1}\left\{\sum_{j=1}^{l_{1}} M_{0}^{(j)}+\sum_{j=1}^{l_{1}} \sum_{p_{j}=0}^{m-2} M_{p_{j}+2}^{(j, i)} \frac{(2 \gamma)^{p_{j}+2}}{\rho^{p_{j}+2}}\right. \\
& \left.+\sum_{j=1}^{l_{2}} \sum_{p_{j}=0}^{m-1} M_{p_{j}+1}^{(j, i)} \frac{(\gamma)^{p_{j}+1}}{\rho^{p_{j}+1}}\right\} \times \frac{(r+k+1+|\nu|) !}{\rho^{|\nu|}},
\end{aligned}
$$

where $M_{\alpha}$ are some constants that are derived from the coefficients of $\mathfrak{M}_{\alpha}$. Setting

$$
C_{1}=\operatorname{Max}\left\{1, \sum_{j=1}^{l_{1}} M_{0}^{(j)}+\sum_{j=1}^{l_{1}} \sum_{p_{j}=0}^{m-2} M_{p_{j}+2}^{(j, i)} \frac{(2 \gamma)^{p_{j}+2}}{\rho^{p_{j}+2}}+\sum_{j=1}^{l_{2}} \sum_{p_{j}=0}^{m-1} M_{p_{j}+1}^{(j, i)} \frac{(\gamma)^{p_{j}+1}}{\rho^{p_{j}+1}}\right\}
$$

that gives a constant independent of $k$, the above inequality becomes

$$
\mid D_{y}^{\nu}\{\text { the right hand side of (2.24) with } k \text { replaced by } k+1\} \mid
$$

$$
\leqslant A B^{k+1} C_{1} \frac{(r+k+1+|\nu|) !}{\rho^{|\nu|}}
$$

so we get the estimates for $U_{-p^{+} h+k+1}^{(i)}(0, y), V_{-p+h+k+1}^{(j)}(0, y)$ and $\frac{\partial U_{-p+h+k}^{(i)}}{\partial t}(0, y)$ :

$$
D_{y}^{\nu}\left\{\begin{array}{l}
U_{-p+h+k+1}^{(i)} \\
V_{-p+h+k+1}^{(j)} \\
\frac{\partial U_{-p+h+k}^{(i)}}{\partial t}
\end{array}\right\}(0, y)\left|\leqslant A B^{k+1} C_{1} \frac{(r+k+1+|\nu|) !}{\rho^{|\nu|}}\right|
$$

Next, let us go to estimates for $U_{-p+h+k}^{(i)}(t, y)$ and $V_{-p+h+k}^{(j)}(t, y)$. In order to estimate $U_{-p+h+k}^{(i)}(t, y)$, we split $U_{-p+h+k}^{(i)}$ in a sum

$$
U_{-p+h+k}^{(i)}=\widehat{U_{-p+h+k}^{(i)}} \curvearrowright \widetilde{\overbrace{}}
$$

where $U_{-p+h+k}^{(i)}$ is the solution of the equation

$$
\begin{aligned}
& \left\{\left(\frac{\partial}{\partial t}\right)^{2}+a_{i}(t, y) \frac{\partial}{\partial t}+b_{i}(t, y)\right\} \widetilde{U_{-p+h+k}^{(i)}} \\
& \quad=\sum_{\alpha=0}^{m-3} \mathscr{L}_{\alpha+3}\left[U_{-p+h+k-1-\alpha}^{(i)}\right]
\end{aligned}
$$


with zero initial data, and $\widetilde{U_{-p+h+k}^{(i)}}$ is defined by the solution of the equation

$$
\left\{\left(\frac{\partial}{\partial t}\right)^{2}+a_{i}(t, y) \frac{\partial}{\partial t}+b_{i}(t, y)\right\} \widetilde{U_{-p+h+k}^{(i)}}=0
$$

with the initial conditions $U_{-p+h+k}^{(i)}(0, y), \frac{\partial U_{-p+h+k}^{(i)}}{\partial t}(0, y) . \widetilde{U_{-p+h+k}^{(i)}}$ is estimated by applying Proposition 2 to (3.8). In order to do so, we must estimate the right hand side of (3.8). From the inductive hypothesis and Proposition 1, it follows that

$$
\begin{aligned}
\mid D_{y}^{\nu} D_{t}^{q} & \sum_{\alpha=0}^{m-3} \mathscr{L}_{\alpha+3}\left\lceil U_{-p+h+k-\alpha-1}^{(i)}\right] \mid \\
\leqslant & \sum_{\alpha=0}^{m-3} L_{\alpha+3} A B^{k-\alpha+1} \frac{(r+k+|\nu|+q+2) !}{\rho^{|\nu|+q+\alpha+3}} \\
& \times \exp (2 \gamma|t|) K(|t|)^{r+k+|\nu|+q+\alpha+3}(2 \gamma)^{\alpha+3+q} \\
\leqslant & \left\{\sum_{\alpha=0}^{m-3} L_{\alpha+3} \frac{1}{\rho^{\alpha+3}} K(1)^{\alpha+1}(2 \gamma)^{\alpha+3}\right\} A B^{k+1} \frac{(r+k+|\nu|+q+2) !}{\rho^{|\nu|+q}} \\
& \times \exp (2 \gamma|t|) K(|t|)^{r+k+|\nu|+q+2}(2 \gamma)^{q} \quad \text { for } \quad|t| \leqslant 1,
\end{aligned}
$$

where $L_{\alpha}$ are some constants depending only on $\mathscr{L}_{\alpha}$. If we put $C_{2}=\sum_{\alpha=0}^{m-3} L_{\alpha} \frac{1}{\rho^{\alpha+3}} K(1)^{\alpha+1}(2 \gamma)^{\alpha+3}$ that is independent of $k$, the above inequality reduces to

$$
\begin{aligned}
& \left|D_{y}^{\nu} D_{t}^{q} \sum_{\alpha=0}^{m-3} \mathscr{L}_{\alpha+3}\left[U_{-p+h+k-1-\alpha}^{(i)}\right]\right| \\
& \quad \leqslant C_{2} A B^{k+1} \frac{(r+k+|\nu|+q+2) !}{\rho^{|\nu|+q}} \exp (2 \gamma|t|) K(|t|)^{r+k+|\nu|+q+2}(2 \gamma)^{q} .
\end{aligned}
$$

This makes it possible to apply Proposition 2 to (3.8) and so we get

$$
\begin{aligned}
& \left|D_{t}^{q} D_{y}^{\nu} \widetilde{U_{-p+h+k}^{(i)}}(t, y)\right| \\
& \leqslant A B^{k+1} C_{2} 2^{2} K(1)^{2} \frac{(r+k+|\nu|+q) !}{\rho^{|\nu|+q}} \\
& \quad \times \exp (2 \gamma|t|) K(|t|)^{r+k+|\nu|+q}(2 \gamma)^{q} .
\end{aligned}
$$


Next, we go to $U_{-p+h+k}$. The initial data $U_{-p+h+k}^{(i)}(0, y), \frac{\partial U_{-p+h+k}^{(i)}}{\partial t}(0, y)$ in the equation (3.9) have been already estimated by $\left(I_{k}\right)$ and (3.7);

$$
\begin{aligned}
& \left|D_{y}^{\nu} U_{-p+h+k}^{(i)}(0, y)\right| \leqslant B^{k+1} A \frac{(r+k+|\nu|) !}{\rho^{|\nu|}} \\
& \left|D_{y}^{\nu} \frac{\partial U_{-p+h+k}^{(i)}}{\partial t}(0, y)\right| \leqslant B^{k+1} A C_{1} \frac{(r+k+1+|\nu|) !}{\rho^{|\nu|}} .
\end{aligned}
$$

Hence, an application of Proposition 3 to (3.9) leads immediately to

$$
\begin{aligned}
& \left|D_{y}^{\nu} D_{t}^{q} U_{-p+h+k}^{(i)}(t, y)\right| \\
& \leqslant 2^{2} B^{k+1} A C_{1} \frac{(r+k+q+|\nu|) !}{\rho^{q+|\nu|}} \exp (2 \gamma|t|) K(|t|)^{r+k+1+q+|\nu|}(2 \gamma)^{q} .
\end{aligned}
$$

Since $U_{-p+h+k}^{(i)}=U_{-p+h+k}^{(i)}+U_{-p+h+k}^{(i)}$, adding (3.10) and (3.11), we have

$$
\begin{aligned}
& \left|D_{t}^{q} D_{y}^{\nu} U_{-p+h+k}^{(i)}(t, y)\right| \\
& \leqslant B^{k+1} A\left[C_{1} K(1)+C_{2} K(1)^{2}\right] 2^{2} \\
& \quad \times \frac{(r+k+q+|\nu|) !}{\rho^{q+|\nu|}} \exp (2 \gamma|t|) K(|t|)^{r+k+q+|\nu|}(2 \gamma)^{q} .
\end{aligned}
$$

By quite the same procedure as $U_{-p+h+k}^{(i)}$, we can estimate $V_{-p+h+k+1}^{(j)}$. Namely, we set

$$
V_{-p+h+k+1}^{(j)}=\dot{V}_{-p+h+k+1}^{(j)}+\stackrel{\sim}{V_{-p+h+k+1}^{(j)}}=
$$

where $\widehat{V_{-p+h+k+1}^{(j)}}$ is the solution of the equation

$$
\left[\frac{\partial}{\partial t}+c_{j}(t, y)\right] \bar{V}_{-p+h+k+1}^{(j)}(t, y)=\sum_{\alpha=0}^{m-2} \mathscr{L}_{\alpha+2}\left[V_{-p+h+k-\alpha}^{(j)}\right]
$$

with zero initial data, and $V_{-p+h+k+1}^{(j)}$ is defined by the solution of the equation

$$
\left[\frac{\partial}{\partial t}+c_{j}(t, y)\right] \widetilde{V_{-p+h+k+1}^{(j)}}(t, y)=0
$$


with initial data $V_{-p+h+k+1}^{(j)}(0, y)$. The right hand side of (3.13) is bounded by

$$
\begin{aligned}
& \left|D_{y}^{\nu} D_{t}^{q} \sum_{\alpha=0}^{m-2} \mathscr{L}_{\alpha+2}\left[V_{-p+h+k-\alpha}^{(j)}\right]\right| \\
& \leqslant \sum_{\alpha=0}^{m-2} A B^{k-\alpha+1} L_{\alpha+2} \frac{(r+k+|\nu|+q+2) !}{\rho^{|\nu|+q+\alpha+2}} \\
& \quad \times \exp (\gamma|t|) K(|t|)^{r+k+|\nu|+q+2}(\gamma)^{q+\alpha+2} \\
& \leqslant B^{k+1} A\left(\sum_{\alpha=0}^{m-2} L_{\alpha+2} \frac{(\gamma)^{q+\alpha+2}}{\rho^{\alpha+2}}\right) \frac{(r+k+|\nu|+q+2) !}{\rho^{|\nu|+q}} \\
& \quad \times \exp (\gamma|t|) K(|t|)^{r+k+2+|\nu|+q}(\gamma)^{q} .
\end{aligned}
$$

If we put

$$
C_{3}=\sum_{\alpha=0}^{m-2} L_{\alpha+2} \frac{(\gamma)^{q+\alpha+2}}{\rho^{\alpha+2}}
$$

that is independent of $k$, the above inequality reduces to

$$
\begin{aligned}
& \left|D_{t}^{q} D_{y}^{\nu} \sum_{\alpha=0}^{m-2} \mathscr{L}_{\alpha+2}\left[V_{-p+h+k-\alpha}^{(j)}\right]\right| \\
& \quad \leqslant A B^{k+1} C_{3} \frac{(r+k+|\nu|+q+2) !}{\rho^{|\nu|+q}} \exp (\gamma|t|) K(|t|)^{r+k+2+|\nu|+q}(\gamma)^{q} .
\end{aligned}
$$

Therefore, applying Proposition 2 to (3.13), we get

$$
\begin{aligned}
& \left|D_{t}^{q} D_{y}^{\nu} \widetilde{V}_{-p+h+k+1}^{(j)}(t, y)\right| \\
& \leqslant 2 B^{k+1} A C_{3} K(1) \frac{(r+k+|\nu|+q+1) !}{\rho^{q+|\nu|}} \\
& \quad \times \exp (\gamma|t|) K(|t|)^{r+k+1+q+|\nu|}(\gamma)^{q} .
\end{aligned}
$$

Next, by (3.7) we have

$$
\left|D_{y}^{\nu} V_{-p+h+k+1}^{(j)}(0, y)\right| \leqslant B^{k+1} A C_{1} \frac{(r+k+1+|\nu|) !}{\rho^{|\nu|}} .
$$

This allows us to apply Proposition 3 to (3.14), and so we get

$$
\left|D_{t}^{q} D_{y}^{\nu} V_{-p+h+k+1}^{(j)}(t, y)\right|
$$




$$
\leqslant 2 B^{k+1} A C_{1} \frac{(r+k+1+q+|\nu|) !}{\rho^{q+|\nu|}} \exp (\gamma|t|) K(|t|)^{r+k+1+q+|\nu|}(\gamma)^{q} .
$$

Adding (3.15) and (3.16), we find that

$$
\begin{aligned}
& \left|D_{t}^{q} D_{y}^{\nu} V_{-p+h+k+1}^{(j)}(t, y)\right| \\
& \leqslant 2 A B^{k+1}\left(C_{3} K(1)+C_{1}\right) \frac{(r+k+1+q+|\nu|) !}{\rho^{q+|\nu|}} \\
& \quad \times \exp (\gamma|t|) K(|t|)^{r+k+1+q+|\nu|}(\gamma)^{q} .
\end{aligned}
$$

Consequently, if we choose for $B$ a constant such that

$$
B=\operatorname{Max}\left\{1,2^{3} E, 2^{2}\left(C_{1} K(1)+C_{2} K(1)^{2}\right), 2\left(C_{3} K(1)+C_{1}\right)\right\},
$$

we can conclude from (3.7), (3.12) and (3.17) that $\left(P_{k+1}\right),\left(Q_{k+1}\right)$ and $\left(I_{k+1}\right)$ remain also valid. Thus, we have completed our induction.

What remains is to prove the exactness of the formal solution (2.4). This follows immediately from the estimates $\left(P_{k}\right)$ and $\left(Q_{k}\right)$. Indeed, $\left(P_{k}\right)$ and $\left(Q_{k}\right)$ lead to the estimates for $u_{-p+h+k+1}^{(i)}(x)$ and $v_{-p+h+k}^{(j)}(x)$ as follows: for $k \geq 0$ and $|x|<\delta$,

$$
\begin{aligned}
& \left|u_{-p+h+k-1}^{(i)}(x)\right| \leqslant A B^{k+1}(r+k-1) ! \exp (2 \gamma \delta) K(\delta)^{r+k} \\
& \left|v_{-p+h+k}^{(j)}(x)\right| \leqslant A B^{k+1}(r+k) ! \exp (\gamma \delta) K(\delta)^{r+k} .
\end{aligned}
$$

In order to prove the exactness of (2.4), we see easily that it is sufficient to discuss on convergence of series

$$
\sum_{k=0}^{\infty} \frac{\left[\varphi^{(i)}\right]^{k}}{k !} u_{k}^{(i)}
$$

In view of (3.18), we can estimate its general term by

$$
\frac{\left|\varphi^{(i)}\right|^{k}}{k !} A B^{k+p-h+1}(r+k+p-h+1) ! \exp (2 \gamma \delta) K(\delta)^{r+k+p-h+1} .
$$

Therefore, the above series converges uniformly on a neighborhood $\left\{\left|\varphi^{(i)}\right|<\frac{1}{B K(\delta)}\right\}$ of $x=0$. This completes the proof of our theorem in the case where the initial data have at most poles.

The proof of our theorem in the case where at least one of the initial data has essential singularity is quite parallel to that of [2] and so we 
shall not repeat it.

\section{§ 4. A Remark on the Existence Domain of the Solution}

In [2] and the theorem in this paper, we showed that, under the conditions imposed there, the solution of the Cauchy problem (1.1) can be analytically continued to a neighborhood of $x=0$ except on at most $m$ characteristic surfaces, or its covering space. In this section, we shall give an example that the existence domain of the solution is more restricted.

We consider the Cauchy problem

$$
\left\{\begin{array}{l}
\frac{\partial^{2} u}{\partial x_{1}^{2}}-\frac{\partial^{2} u}{\partial x_{2} \partial x_{3}}=0 \\
u\left(0, x^{\prime}\right)=w_{1}\left(x^{\prime}\right) \\
\frac{\partial u}{\partial x_{1}}\left(0, x^{\prime}\right)=w_{2}\left(x^{\prime}\right)
\end{array}\right.
$$

We have already noted that this operator $\frac{\partial^{2}}{\partial x_{1}^{2}}-\frac{\partial^{2}}{\partial x_{2} \partial x_{3}}$ does not satisfy condition (A). Surfaces $4 x_{2}\left(x_{3}+c\right)-x_{1}^{2}=0$ ( $c$ : arbitrary constant) and $x_{2}=0$ are simple characteristic surfaces for this operator issuing from $x_{1}=x_{2}=0$ and touch one another. Let

$$
\begin{aligned}
& \mathfrak{D}=\left\{x ;|x|<\frac{1}{2},\left|x_{1}^{2}-4 x_{2} x_{3}\right|<4\left|x_{2}\right|\right\} \\
& \mathfrak{F}=\left\{x ;|x|<\frac{1}{2},\left|x_{1}^{2}-4 x_{2} x_{3}\right| \geqq 4\left|x_{2}\right|\right\} \\
& \mathfrak{B}=\left\{x ;|x|<\frac{1}{2},\left|x_{1}^{2}-4 x_{2} x_{3}\right|=4\left|x_{2}\right|\right\} .
\end{aligned}
$$

We shall show that by giving suitable initial data $w_{1}\left(x^{\prime}\right), w_{2}\left(x^{\prime}\right)$, we can find the solution that is holomorphic in $\mathfrak{D}$, but cannot be analytically continued to 5 .

At first we note that (4.1) has the solution

$$
u(x)=\frac{4 \sqrt{x_{3}+c}}{\delta\left\{4 x_{2}\left(x_{3}+c\right)-x_{1}^{2}\right\}}
$$


with initial data

$$
\left\{\begin{array}{l}
w_{1}\left(x^{\prime}\right)=\frac{1}{\delta x_{2} \sqrt{x_{3}+c}} \\
w_{2}\left(x^{\prime}\right)=0
\end{array}\right.
$$

where $\delta$ and $c$ are constants.

Let $Z$ be one dimensional complex plane with coordinate $c$, and $\left\{c_{n}\right\}_{n=1,2, \ldots}$ be a countable dense set on the unit circle $\{c \in Z ;|c|=1\}$ in $Z$. As is easily seen, we can construct successively disks $D_{n}\left(|c| \leqslant r_{n}, r_{n} \uparrow 1\right)$, points $\xi_{n}\left(\left|\xi_{n}\right|<1\right)$ on $Z$ and positive constants $\delta_{n}$ as follows

(i) $D_{n} \uparrow D=\{c \in Z ;|c|<1\}$

(ii) $\xi_{1}, \ldots, \xi_{n-1} \in D_{n}, \xi_{n} \notin D_{n}, \xi_{n}-c_{n} \rightarrow 0(n \rightarrow \infty)$,

$$
\frac{1}{\delta_{n}} \frac{\left|\sqrt{x_{3}+c_{n}}\right|}{\left|c_{n}-c\right|}<\frac{1}{2^{n}} \text { for all } c \in D_{n} \text { and }\left|x_{3}\right|<\frac{1}{2}
$$

$$
\left(\delta_{n}>2^{n}\right)
$$

and

$$
\frac{1}{\delta_{n}} \frac{\left|\sqrt{x_{3}+c_{n}}\right|}{\left|c_{n}-\xi_{n}\right|}>n+1+\sum_{k=1}^{n-1} \frac{\left|\sqrt{x_{3}+c_{k}}\right|}{\delta_{k}\left|c_{k}-\xi_{n}\right|} \quad \text { for }\left|x_{3}\right|<\frac{1}{2}
$$

This is possible, indeed let us put $c_{k}=e^{i \varphi_{k}}(k=1,2, \ldots, n)$ and $\xi_{n}=r e^{i \varphi_{n}}$, then we may choose $r(<1)$ such that

$$
\frac{\left|\sqrt{x_{3}+c_{n}}\right|}{\delta_{n}|1-r|}>n+1+\sum_{k=1}^{n-1} \frac{\sqrt{\frac{3}{2}}}{\delta_{k}\left|\sin \left(\varphi_{n}-\varphi_{k}\right)\right|} .
$$

Now, let

$$
K_{n}=\left\{x ;|x|<\frac{1}{2}, \frac{x_{1}^{2}-4 x_{2} x_{3}}{4 x_{2}} \in D_{n}\right\}
$$

then

$$
\bigcup_{n=1}^{\infty} K_{n}=\mathfrak{D}
$$


With $c_{n}$ and $\delta_{n}$ in (4.2), we put

$$
u\left(x_{1}, x_{2}, x_{3}\right)=\sum_{n=1}^{\infty} \frac{4 \sqrt{x_{3}+c_{n}}}{\delta_{n}\left[4 x_{2}\left(x_{3}+c_{n}\right)-x_{1}^{2}\right]} .
$$

Since $4 x_{2}\left(x_{3}+c_{n}\right)-x_{1}^{2}=4 x_{2}\left(c_{n}-c\right)$ on $4 x_{2}\left(x_{3}+c\right)-x_{1}^{2}=0$, we have by $(4.2)$

$$
\left|\frac{4 \sqrt{x_{3}+c_{n}}}{\delta_{n}\left[4 x_{2}\left(x_{3}+c_{n}\right)-x_{1}^{2}\right]}\right|<\frac{1}{2^{n}\left|x_{2}\right|} \text { on } K_{n} \text {. }
$$

This means that $u(x)$ is holomorphic in $\mathfrak{D}$.

Next, we want to show that $u(x)$ cannot be analytically continued beyond $\mathfrak{B}$. Let us take any point $\zeta$ on $\mathfrak{B}$ and $c$ be the point on the unit circle which corresponds to $x=\zeta$ by $c=\frac{x_{1}^{2}-4 x_{2} x_{3}}{4 x_{2}}$. Then, we can choose a sequence $\left\{c_{n_{k}}\right\}$ and $\left\{\xi_{n_{k}}\right\}$ in (4.2) with $c_{n_{k}} \rightarrow c$ and $\xi_{n_{k}} \rightarrow c$. Let us take the points $\eta_{n_{k}}$ on the surfaces $4 x_{2}\left(x_{3}+\xi_{n_{k}}\right)-x_{1}^{2}=0$ such that $\eta_{n_{k}} \rightarrow \zeta\left(n_{k} \rightarrow \infty\right)$. In fact, if $\zeta=(\alpha, \beta, \gamma)$, we may put $\eta_{n_{k}}=\left(\alpha, \tilde{\beta}_{n_{k}}, \gamma\right)$ with $\tilde{\beta}_{n_{k}}=\frac{\beta(\gamma+c)}{\gamma+\xi_{n_{k}}}$.

Now, since we have

$$
\begin{aligned}
\left|u\left(\eta_{n_{k}}\right)\right| \geqq & \left\{\frac{\left|\sqrt{x_{3}+c_{n_{k}}}\right|}{\delta_{n_{l}}\left|c_{n_{k}}-\xi_{n_{k}}\right|}-\sum_{l=1}^{n_{k}-1} \frac{\left|\sqrt{x_{3}+c_{l}}\right|}{\delta_{l}\left|c_{l}-\xi_{n_{k}}\right|}\right. \\
& \left.-\sum_{l=n_{k}+1}^{\infty} \frac{\left|\sqrt{x_{3}+c_{l}}\right|}{\delta_{l}\left|c_{l}-\xi_{n_{k}}\right|}\right\} \frac{1}{\left|x_{2}\right|} \quad \text { for } \quad x=\eta_{n_{k}}
\end{aligned}
$$

in view of (4.2) we get

$$
\left|u\left(\eta_{n_{k}}\right)\right| \geqq n_{k} \frac{1}{\left|\tilde{\beta}_{n_{k}}\right|}
$$

This means that $u(x)$ cannot be continued beyond $\mathfrak{B}$.

Consequently, we see that the Cauchy problem (4.1) with initial data

$$
\left\{\begin{array}{l}
w_{1}\left(x^{\prime}\right)=\left\{\sum_{n=1}^{\infty} \frac{1}{\delta_{n} \sqrt{x_{3}+c_{n}}}\right\} \frac{1}{x_{2}} \quad \text { for } \quad\left|x^{\prime}\right|<\frac{1}{2} \\
w_{2}\left(x^{\prime}\right)=0
\end{array}\right.
$$


has the solution in the form

$$
u(x)=\sum_{n=1}^{\infty} \frac{4 \sqrt{x_{3}+c_{n}}}{\delta_{n}\left[4 x_{2}\left(x_{3}+c_{n}\right)-x_{1}^{2}\right]}
$$

which is holomorphic in $\mathfrak{D}$, but cannot be analytically continued to $\sqrt{5}$.

In the same way, when the initial data have essential singularities, we can find the solution that is holomorphic in $\mathfrak{D}$, but cannot be continued to (5. In fact, since (4.1) has the solution in the form

$$
g(k, \delta, c ; x)=\frac{1}{\sqrt{x_{3}+c}}\left\{\frac{4\left(x_{3}+c\right)}{\delta\left[4 x_{2}\left(x_{3}+c\right)-x_{1}^{2}\right]}\right\}^{k},
$$

by choosing suitable sequences $\left\{c_{n}\right\}\left(\left|c_{n}\right|=1\right),\left\{\delta_{n}\right\}\left(\delta_{n}>0\right)$ and $\left\{m_{n}\right\}$ $\left(m_{n}\right.$ : integers $\left.>0\right)$, we can find the solution of (4.1) satisfying the above conditions in the form

$$
u(x)=\sum_{n=1}^{\infty} g\left(m_{n}, \delta_{n}, c_{n} ; x\right)
$$

with initial data

$$
\left\{\begin{array}{l}
w_{1}\left(x^{\prime}\right)=\sum_{n=1}^{\infty} \frac{1}{\left(\delta_{n} x_{2}\right)^{m_{n}}} \frac{1}{\sqrt{x_{3}+c_{n}}} \\
w_{2}\left(x^{\prime}\right)=0 .
\end{array}\right.
$$

\section{References}

[ $\left.\mathbf{1}_{\mathbf{r}}^{\mathbf{k}}\right]$ Gårding, L., T. Kotake et J. Leray, Uniformisation et développement asymptotique de la solution du problème de Cauchy linéaire, à données holomorphes, Bull. Soc. Math. France 92 (1962), 263-361.

[2] Hamada, Y., The singularities of the solutions of the Cauchy problem, Publ. RIMS, Kyoto Univ. 5 (1969), 21-40.

[3] Lax, A., On Cauchy's problem for partial differential equation with multiple characteristics, Comm. Pure Appl. Math. 9 (1956), 135-169.

[4] Ludwig, D., Exact and asymptotic solutions of the Cauchy problem, Comm. Pure Appl. Math. 13 (1960), 473-508.

[5] Matsuura, S., On non-strict hyperbolicity, Proceeding of International Conference on Functional Analysis and Related Topics, Tokyo, April 1969.

[6] Mizohata, S., Solutions nulles et solutions non analytiques, J. Math. Kyoto Univ. 1 (1962), 271-302.

[7] Mizohata, S., Analyticity of the fundamental solutions of hyperbolic system, 
J. Math. Kyoto Univ. 1 (1962), 327-355.

[8] Mizohata, S., et Y. Ohya, Sur la condition de E. E. Levi concernant des équations hyperboliqes, Publ. RIMS, Kyoto Univ. Ser. A, 4 (1968), 511-526. 